\section{Laudatio für Prof. Dr. med. Christian Arning}

Sehr geehrte, liebe Kolleginnen und Kollegen,

lieber Christian Arning,

es ist schön, dich heute nicht wie so häufig in offizieller Funktion, sondern als Ehrenmitglied zu begrüßen.

Durch die Verleihung der Ehrenmitgliedschaft sollst du eine Würdigung deiner langjährigen wissenschaftlichen, klinischen, lehrenden und berufspolitischen Betätigung auf dem Feld des medizinischen Ultraschalls erfahren.

Ich war überrascht, als du mir einmal erzählt hast, dass du kein waschechter Hamburger bist, sondern gebürtig aus Wattenscheid stammst, da ich dich immer als waschechten Hamburger wahrgenommen habe. Mag sein, dass dieser Eindruck verstärkt worden ist durch deinen langjährig getragenen Kapitänsbart, eine Gesichtsmode, die auch als Schifferkrause Bekanntheit erlangt hat.

Deine berufliche Laufbahn jedenfalls hast du mit dem Medizinstudium in Hamburg begonnen, einer Stadt, der du bis heute treu geblieben ist. Hervorheben möchte ich deine langjährige Verbundenheit mit zwei Hamburger Traditionskrankenhäusern; als leitender Oberarzt am Klinikum Barmbek und Chefarzt am Klinikum Wandsbek.

In die Zeit von Christian Arnings Tätigkeit in Barmbek fällt der Beginn seines Interesses für den Ultraschall. Es war die Zeit des Übergangs von der reinen Dopplersonografie zunächst zur Duplex- und dann Farbduplexsonografie, so dass sich für ihn mit den aufkommenden neuen Methoden ein neues klinisches Betätigungsfeld, sowie wissenschaftliche Möglichkeiten eröffneten.

Das hat zu zahlreichen wichtigen und teils auch grundlegenden Publikationen geführt. Besondere Erwähnung finden sollen seine wissenschaftlichen Untersuchungen zu den Dissektionen an den hirnversorgenden Arterien, zur Karotidynie, zu duralen AV-Fisteln, sowie natürlich die zahlreichen Arbeiten zur Diagnostik und Behandlung der Karotisstenose.
Seine intensive Beschäftigung zum Thema der Artefakte in der farbkodierten Duplexsonografie führte 1999 zu seiner Habilitation.

Wir verdanken Christian Arning ein wunderbares Lehrbuch zur farbkodierten Duplexsonografie der hirnversorgenden Arterien, das nach wie vor durch seinen Fundus an wunderbarem Bildmaterial besticht. Exemplarisch für seine Freude an der Bilddokumentation sei hier auch seine B-Bildsonografische Erstbeschreibung eines flottierenden Thrombus in der Arteria carotis interna (Journal of Neurology 1988) erwähnt.

Christian Arnings besondere Expertise und sein didaktisches Geschick haben ihn zu einer berufspolitisch engagierten Persönlichkeit, aber auch zu einem äußerst geschätzten klinischen Lehrer werden lassen. So hat er über einen Zeitraum von 29 Jahren die vaskulären Ultraschallkurse an der Hamburger Ärztekammer geleitet (wenn man das in Hochzeitstagen ausdrücken wollte, wäre das immerhin die Samthochzeit mit der Hamburger Ärztekammer).

Besonders erwähnenswert sind auch die Hamburger Neuro-Ultraschalltage, mit dem schönen Akronym HaNUTa, die sich unter seiner Leitung im Lauf der Jahre zur größten neurologischen Ultraschall-Fachtagung in Deutschland entwickelt haben.

Sein Engagement für die DEGUM in gehobener Funktion begann 1992, als er für einen Zeitraum von sechs Jahren das Sprecheramt des Arbeitskreises Gefäßdiagnostik innehatte. Von 1998 bis 2006 gehörte er dem Vorstand der DEGUM an, zunächst als Beisitzer, dann als Sekretär, von 2004 bis 2006 als unser Präsident.

Herr Arning hat in dieser Zeit viel für die DEGUM geleistet, insbesondere waren ihm die Öffentlichkeitsarbeit und die Außendarstellung gegenüber anderen Fachgesellschaften, sowie die Qualitätssicherung in der Ultraschallaus- und Weiterbildung ein Herzensanliegen.

Wesentliches hat er hier durch eine verbesserte Verankerung des Ultraschalls in den



Christian Arning Verleihung

Weiterbildungsordnungen der Bundesärztekammer analog DEGUM Stufe 1 erreichen können.

Besonders hervorheben möchte ich aber auch sein Verdienst um eine dauerhafte Qualitätssicherung im vaskulären Ultraschall durch die von ihm federführend geleistete Arbeit an der Revision der DEGUM-Kriterien zur Graduierung der Karotisstenose, die 2012 Eingang in die S3 Leitlinie zur Karotisstenose gefunden haben und über diese Leitlinie von allen beteiligten Fachgesellschaften übernommen wurden. Hier wurde Erhebliches geleistet zur Vereinheitlichung unserer Stenosekriterien und zur Beseitigung eines langjährigen Definitionschaos in Deutschland.

Da ich zu seinen Zeiten der Vorstandsarbeit selber noch mit Studium und Weiterbildung beschäftigt war, habe ich einige seiner Weggefährten zu seinen Wesenszügen befragt. Besonders hervorgehoben wurden:

- Seine ruhige, ausgleichende, aber auch entschiedene Art, Diskussionen zu leiten.

- Sein Bemühen um einen intensiven Austausch zwischen den Fachdisziplinen.

- Sein Engagement für eine gedeihliche Zusammenarbeit im Arbeitskreis.

- Seine bereichernden Tagungen.

- Sein Einsatz in der Fort- und Weiterbildung.

- Sein trockener Humor, der plötzlich zuschlägt. 
Lieber Christian, dein außerordentliches Engagement für berufspolitische Angelegenheiten wird abschließend auch noch einmal deutlich, wenn man einen Blick auf die wichtigsten deiner weiteren aktuellen und früheren ehrenamtlichen Tätigkeiten wirft:

- Mitgliedschaft in der Sonografiekommission der KV Hamburg.

- Die Leitung des Fortbildungsausschusses, die stellvertretende Leitung des Ausschusses Qualitätssicherung der
Ärztekammer Hamburg, die Leitung der Fortbildungsakademie der Ärztekammer und die Schriftleitung des Hamburger Ärzteblatts.

- Mitgliedschaft im Deutschen Senat für ärztliche Fortbildung der Bundesärztekammer Berlin (2015 umbenannt in: Ständige Kommission Fortbildung der Bundesärztekammer).
Und man kann davon ausgehen, dass du dich in all diesen Funktionen stets auch für die Interessen einer anhaltend hohen Qualität in der Ultraschalldiagnostik und Ausbildung in Deutschland einzusetzen weißt.

Lieber Christian, zu deiner Ehrenmitgliedschaft gratulieren wir dir ganz herzlich.

Prof. Dr. med. Max Nedelmann 